\title{
JOURNAL.RU
}

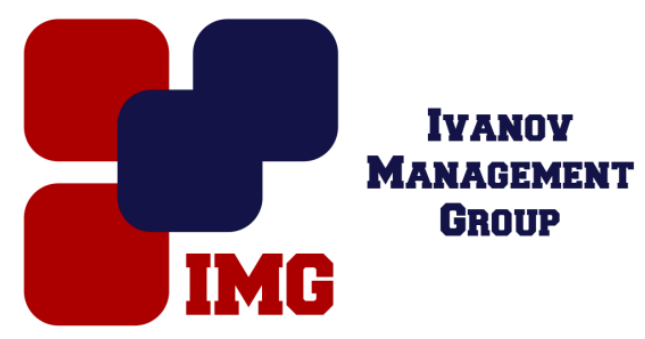

Исмятуллин Р.Г., Мельник С.И., Финогенов С.Н.

Тюменское высшее военно-инженерное командное училище имени маршала инженерных войск А.И. Прошлякова

Тюмень, Россия

doi: $10.18411 / 1 \mathrm{j}-31-07-2017-34$

idsp 000001:1j-31-07-2017-34

\section{Математическая модель оптимального фильтра оценивания промаха и его производных}

\section{Аннотация}

В статье приведен синтез математической модели оптимального фильтра оценивания промаха и его производных, путем определения алгоритма оценивания и характеристик оптимального фильтра.

Ключевые слова: синтез, оптимальный фильтр, расширенная пропорциональная навигация

Математическую постановку задачи синтеза можно сформулировать следующим образом. Для объекта, описываемого уравнением

$$
\ddot{\mathrm{h}} \cong \Delta \mathrm{W}_{\mathrm{y}}=\mathrm{W}_{\mathrm{y} \perp}-\mathrm{W}_{\mathrm{Ly} \perp} \text {. }
$$

необходимо определить алгоритм оценивания и характеристики оптимального фильтра. Полагая движение цели случайным процессом типа белого шума

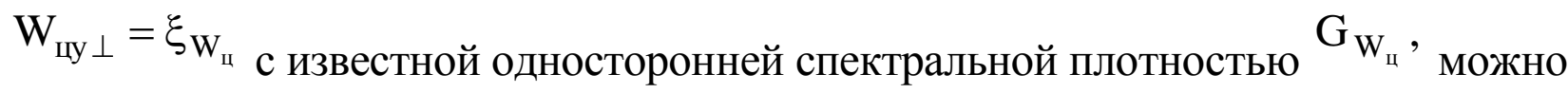
представить обобщенный вектор состояния в виде

$$
\ddot{\mathrm{h}}=-\mathrm{W}_{\mathrm{y} \perp}+\xi_{\mathrm{W}_{\mathrm{u}}} ; \dot{\mathrm{h}}(0)=\dot{\mathrm{h}}_{0} \text {. }
$$

Модель наблюдения имеет вид

$$
\mathrm{Z}=\mathrm{h}+\xi_{\mathrm{h}},
$$

где $\xi \mathrm{h}$ - центрированный белый шум с известной односторонней спектральной плотностью Gh. 
Полагая $\mathrm{X} 1=\mathrm{h}, \mathrm{X}_{2}=\dot{\mathrm{h}}$, модель объекта для случая, когда управление

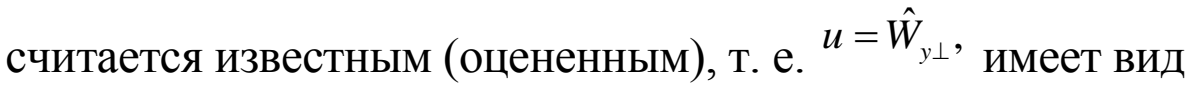

$$
\left.\begin{array}{l}
\dot{\mathrm{X}}_{1}=\mathrm{X}_{2} ; \\
\dot{\mathrm{X}}_{2}=\mathrm{u}+\xi_{\mathrm{W}_{\mathrm{uy}}} \\
\mathrm{Z}=\mathrm{h}+\xi_{\mathrm{h}} .
\end{array}\right\}
$$

Алгоритм оценивания для модели (4) имеет вид

$$
\begin{aligned}
& \left.\begin{array}{l}
\hat{\dot{X}}_{1}=\hat{X}_{2}+K_{\phi 1}\left(Z-\hat{x}_{1}\right) ; \\
\hat{\dot{X}}_{2}=u+K_{\phi 2}\left(Z-\hat{x}_{1}\right) .
\end{array}\right\} \\
& \mathrm{K}_{\phi 1}=\frac{2 \mathrm{D}_{11}}{\mathrm{G}_{\mathrm{h}}} ; \quad \mathrm{K}_{\phi 2}=\frac{2 \mathrm{D}_{12}}{\mathrm{G}_{\mathrm{h}}} ; \\
& \dot{\mathrm{D}}_{11}=2 \mathrm{D}_{12}-\frac{\mathrm{D}_{11}^{2}}{\mathrm{G}_{\mathrm{h}}} ; \quad \mathrm{D}_{11}(0)=\mathrm{D}_{110} ; \\
& \left.\dot{\mathrm{D}}_{12}=2 \mathrm{D}_{22}-\frac{\mathrm{D}_{11} \mathrm{D}_{12}}{\mathrm{G}_{\mathrm{h}}} ; \quad \mathrm{D}_{12}(0)=0 ;\right\} \\
& \dot{\mathrm{D}}_{22}=-\frac{\mathrm{D}_{22}^{2}}{\mathrm{G}_{\mathrm{h}}}+0,5 \mathrm{G}_{\mathrm{W}_{\mathrm{u}}} ; \quad \mathrm{D}_{11}(0)=\mathrm{D}_{110} ;
\end{aligned}
$$

B отличие от дисперсий ошибок оценивания Dij и коэффициентов невязок Кфіј при оценивании Фл, в данном случае отсутствует зависимость от условий наведения, в частности, от $\mathrm{r}_{\text {л }} \dot{\mathrm{r}}_{\text {л }} \cdot$ В предположении постоянства значений спектральных плотностей $\mathrm{G}_{\mathrm{W}_{\text {u }}}$ и $\mathrm{Gh}$ можно определить установившиеся значения Dij и Кфij. Для этого необходимо решить систему алгебраических уравнений:

$$
\left.\begin{array}{l}
2 \mathrm{D}_{12}-\frac{\mathrm{D}_{11}^{2}}{\mathrm{G}_{\mathrm{h}}}=0 ; \\
2 \mathrm{D}_{22}-\frac{\mathrm{D}_{11} \mathrm{D}_{12}}{\mathrm{G}_{\mathrm{h}}}=0 ; \\
-\frac{\mathrm{D}_{22}^{2}}{\mathrm{G}_{\mathrm{h}}}+0,5 \mathrm{G}_{\mathrm{W}_{\text {и }}}=0 .
\end{array}\right\}
$$


В результате имеем

$$
\begin{aligned}
& \mathrm{D}_{11}=\sqrt{2 \mathrm{G}_{\mathrm{h}} \sqrt{0,5 \mathrm{G}_{\mathrm{h}} \mathrm{G}_{\mathrm{W}_{\mathrm{uy}}}}} ; \\
& \mathrm{D}_{12}=\sqrt{0,5 \mathrm{G}_{\mathrm{h}} \mathrm{G}_{\mathrm{W}_{\text {uy }}}} ; \\
& \mathrm{D}_{22}=\sqrt{\mathrm{G}_{\mathrm{W}_{\mathrm{uy}}} \sqrt{0,5 \mathrm{G}_{\mathrm{h}} \mathrm{G}_{\mathrm{W}_{\mathrm{uy}}}}} \cdot
\end{aligned}
$$

Тогда

$$
\left.\begin{array}{l}
\mathrm{K}_{\phi 1}=\frac{2 \mathrm{D}_{11}}{\mathrm{G}_{\mathrm{h}}}=\sqrt{2 \sqrt{0,5 \frac{\mathrm{G}_{\mathrm{W}_{\mathrm{uy}}}}{\mathrm{G}_{\mathrm{h}}}}} ; \\
\mathrm{K}_{\phi 2}=\frac{2 \mathrm{D}_{12}}{\mathrm{G}_{\mathrm{h}}}=\sqrt{0,5 \frac{\mathrm{G}_{\mathrm{W}_{\mathrm{Ly}}}}{\mathrm{G}_{\mathrm{h}}}}
\end{array}\right\}
$$

Структурная схема оптимального фильтра для модели (2), (3) приведена на рис. 1.

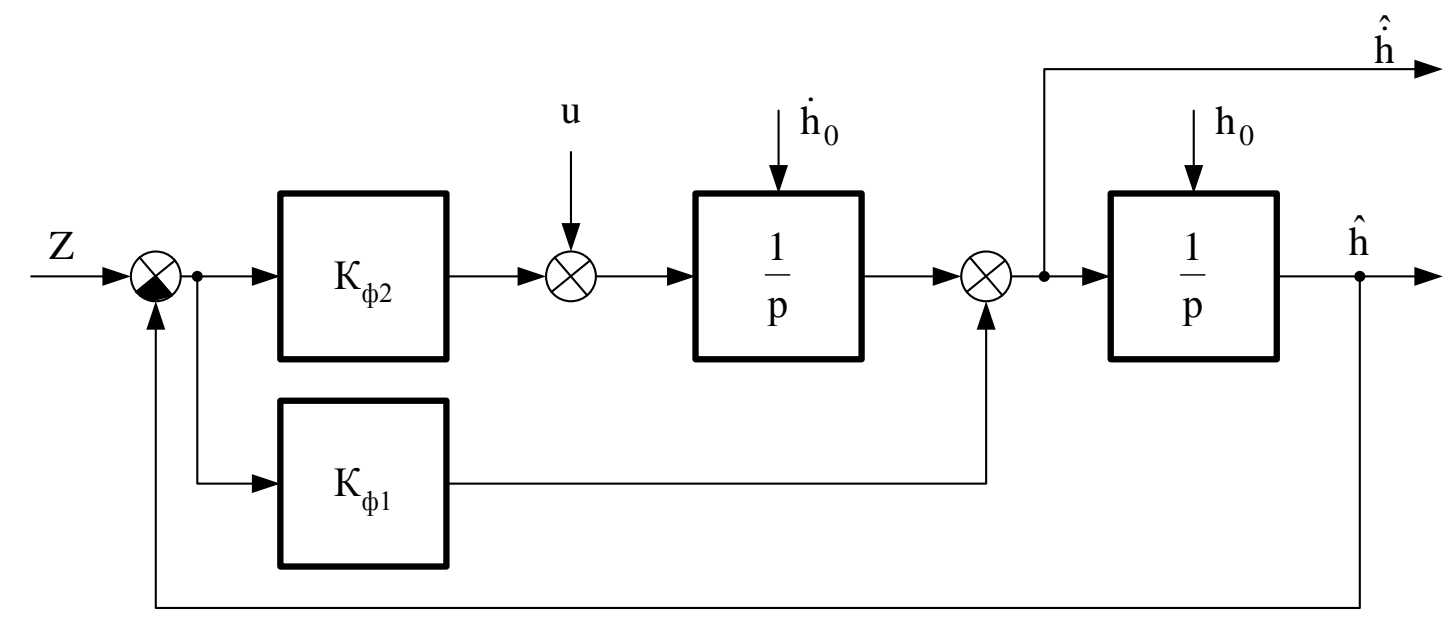

Рис. 1. Структурная схема оптимального фильтра для модели (2) и (3)

Таким образом, для двухмерного фильтра при известном управлении постоянные коэффициенты фильтра соответствуют постоянным интенсивностям шумов объекта и измерений. Двухмерный фильтр дает оптимальные оценки промаха и его производной $\dot{\mathrm{h}}$, использование которых при формировании траекторного управления позволяет повысить точность самонаведения.

Более высокой точности самонаведения, особенно на интенсивно маневрирующие цели, можно достигнуть при увеличении порядка модели до третьего. Это объясняется тем, что более высокие производные лучше 
аппроксимируются некоррелированным шумовым процессом [1]. Кроме того, повышение порядка модели дает возможность получить оптимальные оценки вторых производных параметра управления (ошибок наведения).

В качестве исходной модели воспользуемся выражениями (2), (3).

Полагая $\mathrm{X} 1=\mathrm{h}, \mathrm{X}_{2}=\dot{\mathrm{h}}, \mathrm{X}_{3}=\ddot{\mathrm{h}}$, модель объекта для случая, когда управление считается известным $u=\hat{W}_{y \perp}$, имеет вид

$$
\begin{aligned}
& \dot{\mathbf{X}}=\mathbf{A} \mathbf{X}+\mathbf{B} \mathbf{u}+\xi_{\mathrm{x}} ; \\
& \mathbf{Z}=\mathbf{H X}+\xi_{\mathrm{h}},
\end{aligned}
$$

где

$$
\begin{aligned}
& \mathrm{X}=\left[\begin{array}{lll}
\mathrm{h} & \dot{\mathrm{h}} & \ddot{\mathrm{h}}
\end{array}\right]^{\mathrm{T}} ; \quad \mathrm{u}=\left[\begin{array}{lll}
0 & 1 & 0
\end{array}\right]^{\mathrm{T}} ; \\
& \mathrm{A}=\left[\begin{array}{ccc}
0 & 1 & 0 \\
0 & 0 & 1 \\
0 & 0 & 0
\end{array}\right] ; \quad \mathrm{B}=\left[\begin{array}{l}
0 \\
1 \\
0
\end{array}\right] ; \quad \mathrm{H}=\left[\begin{array}{l}
1 \\
0 \\
0
\end{array}\right]
\end{aligned}
$$

Алгоритм оценивания:

$$
\begin{gathered}
\hat{\dot{X}}_{1}=\hat{X}_{2}+K_{\phi 1}\left(Z-\hat{x}_{1}\right) ; \\
\hat{\dot{X}}_{2}=\hat{X}_{3}+u+K_{\phi 2}\left(Z-\hat{x}_{1}\right) ; \\
\hat{\dot{X}}_{3}=K_{\phi 3}\left(Z-\hat{x}_{1}\right) ; \\
\mathrm{K}_{\phi 1}=\frac{2 \mathrm{D}_{11}}{\mathrm{G}_{\mathrm{h}}} ; \mathrm{K}_{\phi 2}=\frac{2 \mathrm{D}_{21}}{\mathrm{G}_{\mathrm{h}}} ; \mathrm{K}_{\phi 3}=\frac{2 \mathrm{D}_{31}}{\mathrm{G}_{\mathrm{h}}} ;
\end{gathered}
$$



$\dot{\mathrm{D}}_{11}=2 \mathrm{D}_{21}-\frac{\mathrm{D}_{11}^{2}}{\mathrm{G}_{\mathrm{h}}} ;$
$\mathrm{D}_{11}(0)=\mathrm{D}_{110}$
$\dot{\mathrm{D}}_{21}=2 \mathrm{D}_{22}+\mathrm{D}_{21}+\mathrm{D}_{31}-\frac{\mathrm{D}_{21} \mathrm{D}_{11}}{\mathrm{G}_{\mathrm{h}}}$;
$\mathrm{D}_{21}(0)=0$
$\dot{\mathrm{D}}_{22}=2 \mathrm{D}_{32}+2 \mathrm{D}_{22}-\frac{\mathrm{D}_{21}^{2}}{\mathrm{G}_{\mathrm{h}}} ;$
$\mathrm{D}_{22}(0)=\mathrm{D}_{220}$;
$\dot{\mathrm{D}}_{31}=\mathrm{D}_{23}-\frac{\mathrm{D}_{31} \mathrm{D}_{11}}{\mathrm{G}_{\mathrm{h}}} ;$
$\mathrm{D}_{31}(0)=0$
$\dot{\mathrm{D}}_{21}=\mathrm{D}_{33}+\mathrm{D}_{32}-\frac{\mathrm{D}_{31} \mathrm{D}_{21}}{\mathrm{G}_{\mathrm{h}}} ;$
$\mathrm{D}_{32}(0)=0$
$\dot{\mathrm{D}}_{33}=-\frac{\mathrm{D}_{31}^{2}}{\mathrm{G}_{\mathrm{h}}}+0,5 \mathrm{G}_{\mathrm{W}_{\text {ue }}} ;$
$\mathrm{D}_{33}(0)=\mathrm{D}_{330}$.

Структурная схема трехмерного оптимального фильтра представлена на рис. 2.

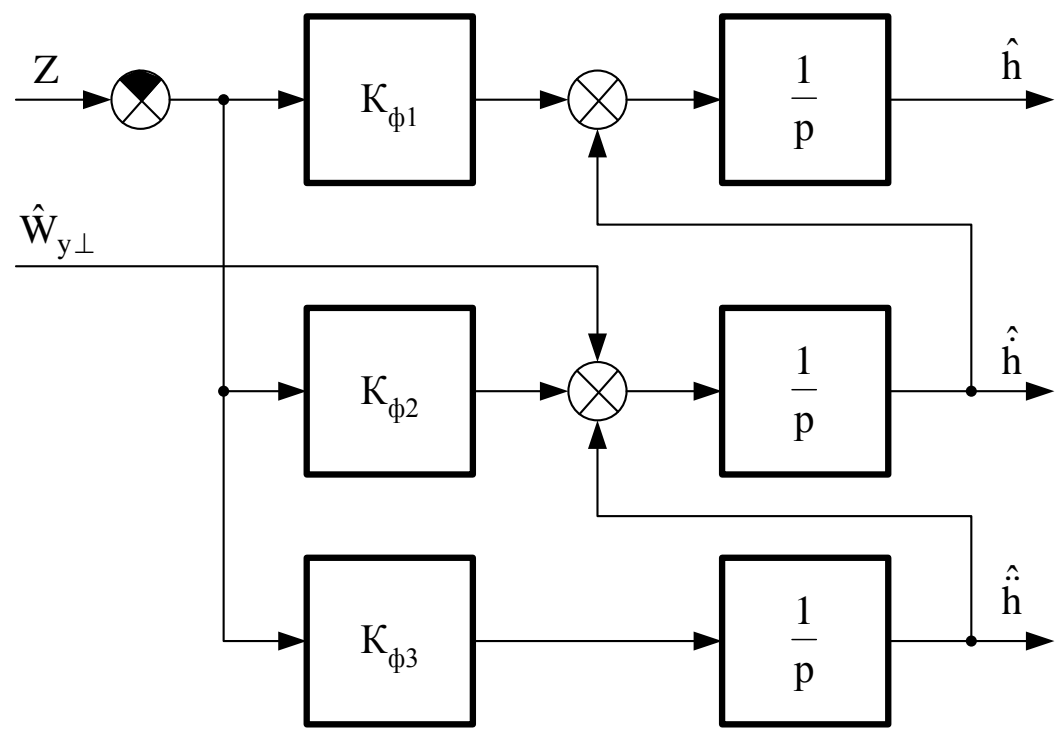

Рис. 2. Структурная схема трехмерного оптимального фильтра

Трехмерный фильтр дает оптимальные оценки параметра управления $\mathrm{h}$ и его производных $\dot{\mathrm{h}}, \ddot{\mathrm{h}}$, использование которых позволяет формировать траекторное управление, обеспечивающее возможность реализовать самонаведение на интенсивно маневрирующие цели. Кроме того, использование трехмерных фильтров позволяет с достаточной точностью экстраполировать кинематические траектории автономного участка полета летательного аппарата при комбинированном наведении с самонаведением на конечном участке перехвата цели. 
В случае предположения о случайном процессе движения цели, описываемом моделью Зингера $[1,2]$, к уравнению модели (2) необходимо добавить уравнение формирующего фильтра

$$
\dot{\mathrm{W}}_{\mathrm{Ly} \perp}=-\alpha_{\mathrm{W}_{\mathrm{u}}} \mathrm{W}_{\mathrm{Ly} \perp}+\xi_{\mathrm{W}_{\mathrm{Ly}}}, \quad \mathrm{W}_{\mathrm{Ly}}(0)=\mathrm{W}_{\mathrm{Ly} 0},
$$

где $\xi_{\mathrm{W}_{\mathrm{uy}}}-$ центрированный белый шум с известной однородной спектральной плотностью $\mathrm{G}_{\mathrm{W}_{\text {цу }}}$.

При этом обобщенный вектор состояния $\mathrm{X}$, вектор наблюдения $\mathrm{Z}$ и матрица А принимают вид

$$
\mathbf{X}=\left[\begin{array}{lll}
\mathrm{h} & \dot{\mathrm{h}} & \mathrm{W}_{\text {цу } \perp}
\end{array}\right]^{\mathrm{T}}, \mathbf{Z}=\mathbf{H} \mathbf{X}+\xi_{\mathrm{W}_{\text {цу }}}, \mathbf{A}=\left[\begin{array}{ccc}
0 & 1 & 0 \\
0 & 0 & 1 \\
0 & 0 & -\alpha_{\mathrm{W}_{\text {и }}}
\end{array}\right] .
$$

С учетом этих изменений алгоритм оценивания приобретает вид

$$
\left.\begin{array}{l}
\hat{\dot{X}}_{1}=\hat{X}_{2}+K_{\phi 1}\left(Z-\hat{x}_{1}\right) ; \\
\hat{\dot{X}}_{2}=\hat{X}_{3}+u+K_{\phi 2}\left(Z-\hat{x}_{1}\right) ; \\
\hat{\dot{X}}_{3}=-\alpha_{W_{4}} \hat{X}_{3}+K_{\phi 3}\left(Z-\hat{x}_{1}\right),
\end{array}\right\}
$$

где Кф1, Кф2, Кф определяются согласно выражениям (12); дисперсии ошибок оценивания D11 - D32 - согласно выражениям (13); D33 определяется решением уравнения Рикатти

$$
\dot{\mathrm{D}}_{33}=-\alpha_{\mathrm{W}_{\mathrm{uy}}} \mathrm{D}_{33}-\frac{\mathrm{D}_{31}^{2}}{\mathrm{G}_{\mathrm{h}}}+0,5 \mathrm{G}_{\mathrm{W}_{\mathrm{uy}}} \text {. }
$$

Структурная схема оптимального трехмерного фильтра представлена на рис. 3. 


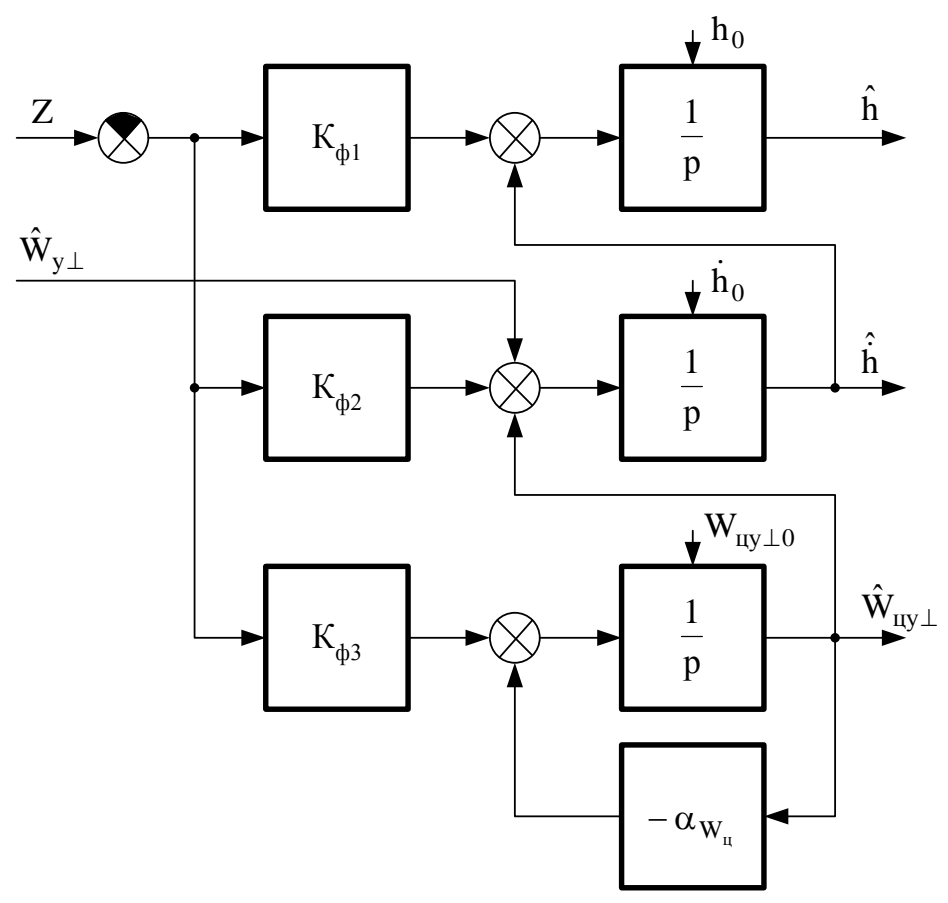

Рис. 3. Структурная схема трехмерного оптимального фильтра с оценкой $\mathbf{W}_{\text {цу } \perp}$

В отличие от оптимального фильтра (рис. 2) данный фильтр позволяет

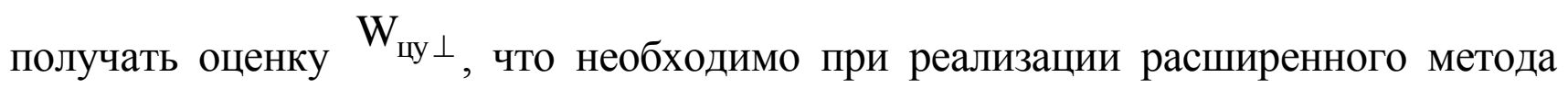
пропорциональной навигации [3].

$* * *$

1. Авиационные системы радиоуправления: Радиоэлектронные системы самонаведения/ Под ред. А. И. Канащенкова и В. И. Меркулова. М., Радиотехника, 2003. 390 с.

2. Зингер Р. А. Оценка характеристик оптимального фильтра для слежения за пилотируемой целью// Зарубежная радиоэлектроника. 1971. № 8.

3. Орлов В. Е. Проектирование систем телеуправления. Ижевск, Изд. дом «Удмуртский ун$\mathrm{T»}, 2000.272 \mathrm{c}$.

4. Финогенов С.Н., Хуторской И.Н., Жарков С.В. Системы самонаведения зенитных ракет. Смоленск, ОАО «Алина», 2006.

5. Финогенов С.Н., Хуторской И.Н. Системы наведения зенитных ракет с оптимальным управлением. Смоленск, ВА ВПВО ВС РФ, 2009.

6. Финогенов С.Н., Царьков М.Е., Або Хамра В. Алгоритм оптимального траекторного управления летательным аппаратом. Москва, Радиотехника. «Нелинейный мир». № 4, 2013. 